\title{
On the Statistical Properties of Ergodic Economic Systems
}

\author{
WEIHONG HUANG ${ }^{\mathrm{a}, *}$ and RICHARD H. DAY ${ }^{\mathrm{b}, \dagger}$ \\ ${ }^{a}$ Nanyang Business School, Nanyang Technological University, Nanyang Avenue, Singapore 639798; \\ ${ }^{\mathrm{b}}$ Department of Economics, University of Southern California, 3620 S. Vermont Avenue, \\ Los Angeles, CA 90089-0253, USA \\ (Received 7 May 2000)
}

Keywords: Chaos; Statistical dynamics; Nonlinear dynamics; Ergodic systems; Tatonnement

\section{MOTIVATIONS}

While chaotic economical systems have been intensively studied over the last two decades, their statistical properties in the long-run, especially their relations to ergodic dynamical systems, did not arouse enough attention from economists. Limited studies were mainly performed by the present authors (see references).

Chaotic economic processes are characterized by sensitive dependence to initial conditions, observation and measurement accuracy, and computation errors, which makes long-term prediction of their trajectories impossible. On the other hand, if a chaotic process preserves an invariant measure, that is, a probability density over its domain, its long-term statistical behavior is robust to the factors mentioned above. Especially when such a chaotic process is also ergodic, the time averages of any continuous function of system's variables exist and can be obtained by the space averages calculated with the invariant density.

In this paper, we reveal some important statistical properties of ergodic processes, which have never been discussed in the literature. These properties not only help us to understand better the internal mechanism of an ergodic process but also provide new insights about dynamical economic processes. It is found that, for a broader class of one-dimensional, discrete time process widely applied in economics, the average growth rate of an ergodic process is always positive, regardless of the explicit form of the process.

Section 2 briefly summarizes the characteristics of an ergodic process. Section 3 introduces an interesting statistical inequality that follows directly from the relevant definitions but has never been pointed out. Section 4 turns to discussion of the growth rate of an ergodic process. Section 5 provides an economic application to Walras'

\footnotetext{
* Corresponding author. e-mail: awhhuang@ntu.edu.sg

†e-mail: rday@rcf.usc.edu
} 
tatonnement process. Section 6 concludes the paper.

\section{CHAOTIC AND ERGODIC PROCESSES}

Let $x_{t+1}=\theta\left(x_{t}\right)$ be an one-dimensional dynamical process defined on a domain $I=[a, b]$. Under some rather weak mathematical requirements such as period three, the process will exhibit chaos, that is, perpetual and erratically fluctuations. Although trajectories that start with two adjacent values will diverge so as to become totally uncorrelated, the frequencies of trajectory $\left\{x_{t}\right\}_{t=0}^{\infty}$ may converge to a unique stable density function, say $\varphi(x)$, which enjoys the same properties as that of a stochastic process, i.e., $\varphi(x) \geq 0$ for all $x$ and $\int_{I} \varphi(x) d x=1$. Due to the sensitive dependence on the initial condition, it is meaningless, in some situations, to study an individual trajectory $\left\{x_{t}\right\}_{t=0}^{\infty}$. Instead, we are more concerned with the long-term characteristics such as the observed density given by $\lim _{T \rightarrow \infty}(1 / T) \sum_{t=0}^{T-1} \chi_{A}\left(\theta^{t}\left(x_{0}\right)\right)$ for any subset $A$ of $I$, the time-average given by

$$
\langle x\rangle=\lim _{T \rightarrow \infty} \frac{1}{T} \sum_{t=0}^{T-1} x_{t}=\lim _{T \rightarrow \infty} \frac{1}{T} \sum_{t=0}^{T-1} \theta^{t}\left(x_{0}\right),
$$

and other statistical characteristics, where $\chi_{A}(\cdot)$ denotes the characteristic function of $A$.

As Lorenz once pointed out, individual trajectories are analogous to weather, while long-term global properties such as invariant density are analogous to climate (Lorenz, 1993). Long-term dynamical properties reveal the whole picture of dynamical process with much richer information that is difficult to attain from individual trajectory. Studies of long-term statistical characteristics can be simplified if the chaotic process is also an ergodic one (its asymptotical behavior can not be studies in a sub-space).

It is clear to mathematicians now that a chaotic dynamical process is also an ergodic process if and only if the invariant measure preserved by it is unique and absolutely continuous with respect to the Lebesgue measure. Hence, through the invariant measure (density), the two conceptually different classes of dynamical characteristics are linked together so that the long-term behavior of a chaotic dynamical process can be investigated by virtue of the fruitful results available in the field of stochastic process (Lasota and Mackey, 1985).

An ergodic process $\theta$ enjoys the following nice properties:

(i) Ergodicity: for any continuous function of $x$, its space average equals to the time average. That is, the following identity holds for any $f \in C^{1}$ and all most all $x_{0} \in I$ :

$$
\langle f(x)\rangle=\lim _{T \rightarrow \infty} \frac{1}{T} \sum_{t=0}^{T-1} f\left(\theta^{t}\left(x_{0}\right)\right)=\int_{I} f(x) \varphi(x) d x,
$$

where $\varphi(x)$ is the invariant density function.

(ii) Stochasticity: the time average will obey the central limit theorem so that the observed trajectory $\left\{x_{t}\right\}_{t=0}^{\infty}$ behaves like a stochastic process.

(iii) Invariance: the time average is invariant with system's iterates, that is, if $\theta$ is an ergodic process, then for any $f \in C^{1}$ and positive integer $k$, the following identities hold true:

$$
\left\langle f\left(\theta^{k}(x)\right)\right\rangle=\langle f(x)\rangle=\left\langle f\left(\theta^{-k}(x)\right)\right\rangle .
$$

Property (i) implies that the time-average of any statistical property is independent of trajectory (initial point) so $\left\langle f\left(x_{0}\right)\right\rangle$ can be written as $\langle f(x)\rangle$.

When $f \equiv x$, the identities (1) become

$$
\left\langle\theta^{+k}(x)\right\rangle=\langle x\rangle, \text { for all positive integers } k .
$$

\section{AN INTERESTING STATISTICAL INEQUALITY}

With identity (2) in hand, we wonder how $\langle x \theta(x)\rangle$ behaves in general? Does there exist any qualitative relationship between $\langle x \theta(x)\rangle$ and $\left\langle x^{2}\right\rangle$ ? Actually, we have 
Proposition 1 For any ergodic dynamical process $x_{t+1}=\theta\left(x_{t}\right)$, there exists the following inequality:

$$
\left\langle x^{m}(\theta(x))^{m}\right\rangle<\left\langle x^{2 m}\right\rangle
$$

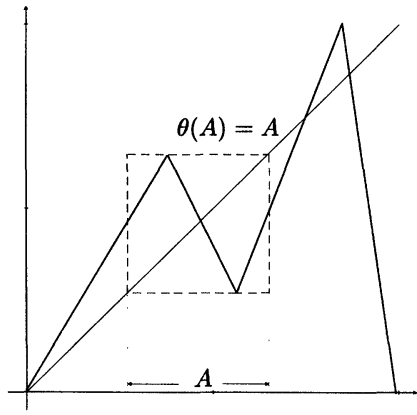

FIGURE 1 Illustration of chaotic but non-ergodic processes.

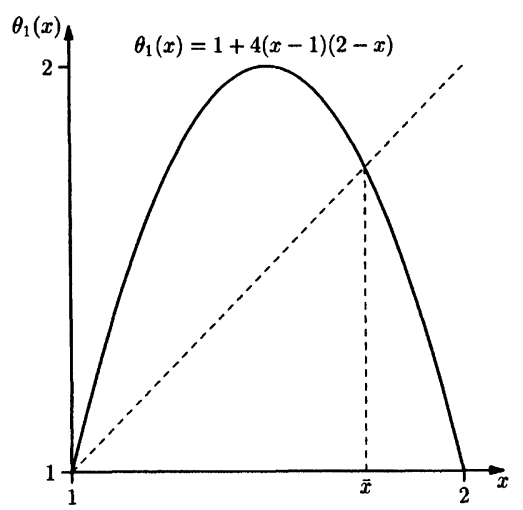

(a). $\theta_{1}(x)=1+4(x-1)(2-x)$

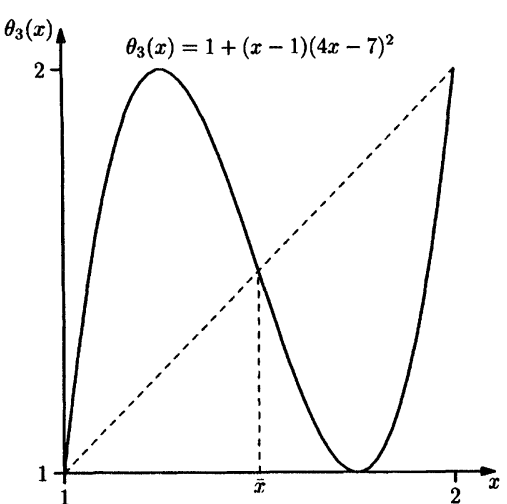

(c). $\theta_{3}(x)=1+(x-1)(4 x-7)^{2}$

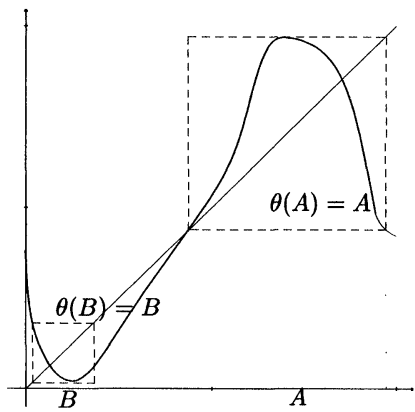

where $m$ is positive constant. If $\theta(x) \neq 0$ for all $x$ in the domain, then the inequality will hold when $m$ takes negative value.

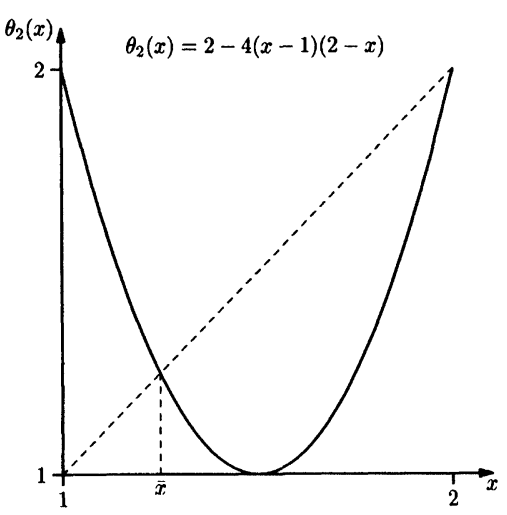

(b). $\theta_{2}(x)=2-4(x-1)(2-x)$

$\theta_{4}(x) \quad \theta_{4}(x)=\theta_{1}(x) \chi_{[1,3 / 2)}+\theta_{2}(x) \chi_{[3 / 2,1]}$

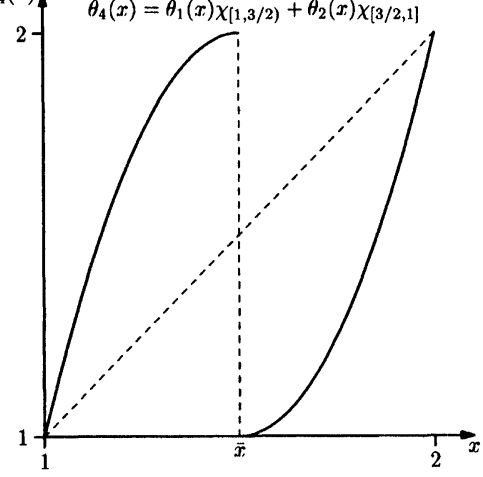

(d).

FIGURE 2 Ergodic processes. 
Proof The proof is straightforward. If $\theta(x)$ is an ergodic process, its time-average can be defined. It follows from the inequality $\left(x^{m}-(\theta(x))^{m}\right)^{2} \geq 0$ that

$$
\left\langle\left(x^{m}-(\theta(x))^{m}\right)^{2}\right\rangle>0
$$

where strict inequality holds because $\theta\left(x_{t}\right) \neq x_{t}$ in general. Inequality (4) in turn implies that

$$
\left\langle x^{2 m}\right\rangle+\left\langle(\theta(x))^{2 m}\right\rangle>2\left\langle x^{m}(\theta(x))^{m}\right\rangle,
$$

which yields the inequality (3) because $\left\langle x^{2 m}\right\rangle=\left\langle(\theta(x))^{2 m}\right\rangle$.

Q.E.D.

Although inequality (3) seems trivial both in the form and in its proof, it does not hold for a chaotic process in general, since the existence of timeaverage of the later may not be guaranteed (Sigmund, 1992). Figure 1 demonstrates two examples of chaotic but non-ergodic processes.
Example 1

$$
\begin{gathered}
\theta_{1}(x)=1+4(x-1)(2-x) \\
\theta_{2}(x)=2-4(x-1)(2-x) \\
\theta_{3}(x)=1+(x-1)(4 x-7)^{2} \\
\theta_{4}(x)=\theta_{1}(x) \chi_{[1,3 / 2)}+\theta_{2}(x) \chi_{[3 / 2,2]}
\end{gathered}
$$

These processes, illustrated in Figures $2 \mathrm{a}-2 \mathrm{~d}$, are among the most frequently discussed chaotic maps in the economic literature. Figure 3 demonstrate the computer simulations of time averages under the same initial condition $x_{0}=1.3333$ for initial 1000 iterations. We see that, for all $i$, we have $\left\langle x \theta_{i}(x)\right\rangle\left\langle\left\langle x^{2}\right\rangle\right.$ and $\left\langle\left(1 / x \theta_{i}(x)\right)\right\rangle\left\langle\left\langle\left(1 / x^{2}\right)\right\rangle\right.$. It can be shown that chaotic processes listed in Example 1 share the same invariant density:
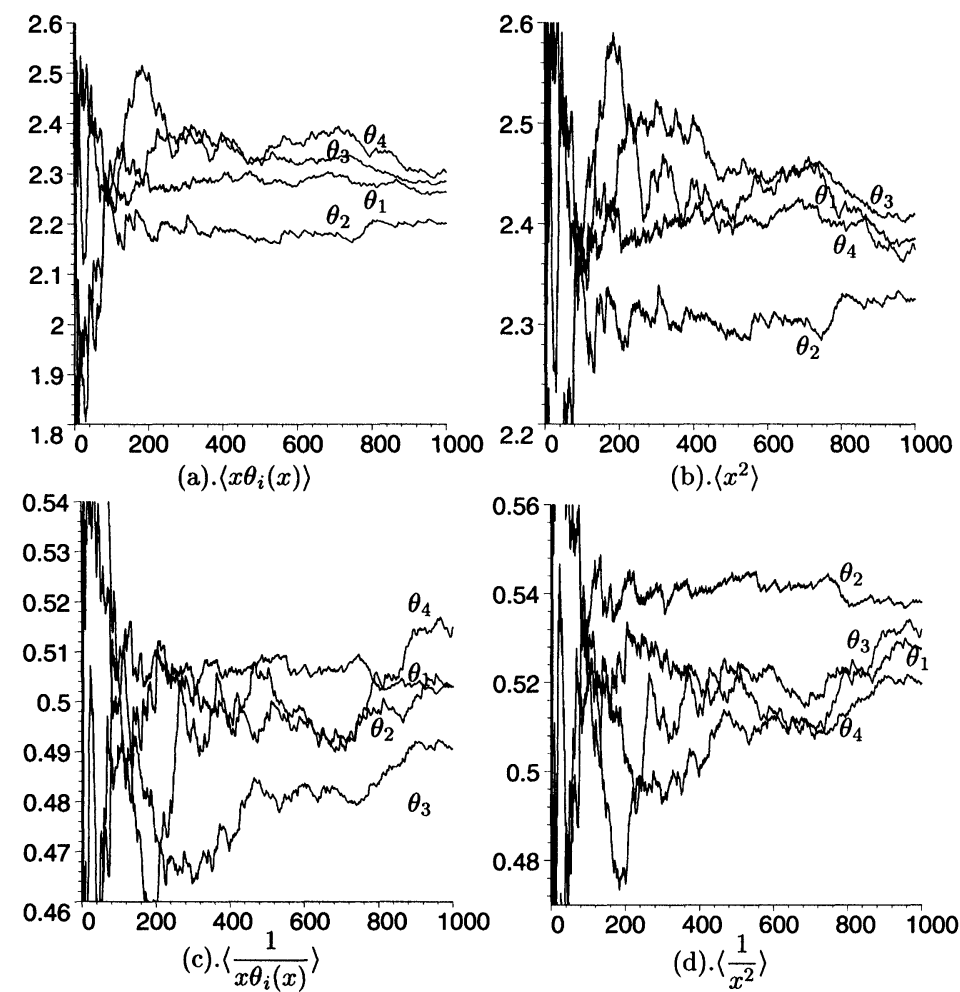

FIGURE 3 Computer simulations I. 
TABLE I Illustration of inequality (3)

\begin{tabular}{lllll}
\hline$i$ & \multicolumn{1}{c}{1} & \multicolumn{1}{c}{2} & \multicolumn{1}{c}{3} & \multicolumn{1}{c}{4} \\
\hline $\int_{I} x \theta_{i}(x) \varphi(x) d x$ & 2.25 & 2.25 & 2.25 & 2.3030 \\
$\int_{I}\left(\varphi(x) / x \theta_{i}(x)\right) d x$ & 0.5051 & 0.4950 & 0.5009 & 0.5132 \\
\hline
\end{tabular}

$\varphi(x)=(1 / \pi \sqrt{(x-1)(2-x)})$. So we can verify the inequalities with space averages, the results of which are summarized in the Table I below.

\section{GROWTH RATE OF DYNAMICAL PROCESS}

Economic growth rate and price inflation rate are two most important concepts in economics, so are income dynamics and price dynamics. Generally, for a dynamical process $x_{t+1}=\theta\left(x_{t}\right)$, if $x_{t} \in R^{+}$, we define the (forward) growth rate as

$$
\begin{aligned}
g_{t} & =\frac{x_{t+1}-x_{t}}{x_{t}} \\
& =\frac{\theta\left(x_{t}\right)}{x_{t}}-1
\end{aligned}
$$

Of course, we can similarly define a backward growth rate as

$$
\begin{aligned}
\tilde{g}_{t} & =\frac{x_{t}-x_{t+1}}{x_{t+1}} \\
& =\frac{x_{t}}{\theta\left(x_{t}\right)}-1
\end{aligned}
$$

Then the following inequality holds for any process:

$$
g_{t} \tilde{g}_{t}<0, \quad \text { for all } t \text {. }
$$

If the trajectory of the process $\theta\left(x_{t}\right)$ is monotonic in the long-run, that is, there exists a time $t^{*}$ such that either $\theta\left(x_{t}\right) \gtrless x_{t}$, for all $t>t^{*}$. So we have $g_{t} \gtrless 0$, for all $t>t^{*}$. So the time-average of $g_{t}$, denoted as $\left\langle g_{t}\right\rangle$, may not exist.

If the trajectory of the process $\theta\left(x_{t}\right)$ is periodic in the long-run, that is, there exists a time $t^{*}$ and positive integer $k$ such that $\theta\left(x_{t+k}\right)=x_{k}$, for all $t>t^{*}$, then the growth rate $g_{t}$ also fluctuates cyclically. So long as the period $k$ is finitely large, the time-averages of $g_{t}$ and $\tilde{g}_{t}$, in contrast to the monotonic case, do exist.

But if $\theta\left(x_{t}\right)$ is chaotic, the growth rate $g_{t}$ will fluctuate irregularly as does the inverse growth rate $\tilde{g}_{t}$. Numerical computer simulations, however, show a paradoxical phenomenon: $\left\langle g_{t}\right\rangle>0$ and $\left\langle\tilde{g}_{t}\right\rangle>0$, that is, both the forward and backward growth rates are always positive, which seemingly contradicts the inequality (12), a characteristic that should be obeyed by all dynamical Process.

Actually, $\left\langle g_{t}\right\rangle>0$ and $\left\langle\tilde{g}_{t}\right\rangle>0$ amount to the concurrent existence of the inequalities:

$$
\left\langle\frac{\theta(x)}{x}\right\rangle>1 \text { and }\left\langle\frac{x}{\theta(x)}\right\rangle>1 \text {. }
$$

Figure 4 demonstrates the computer simulations for the process defined in Example 1 with the same initial condition $x_{0}=1.3333$ for initial 1000 iterations. We see that, for all $i=1,2, \ldots, 4$, we have $\left\langle\left(\theta_{i}(x) / x\right)\right\rangle>1$ and $\left\langle\left(x / \theta_{i}(x)\right)\right\rangle>1$. This can be verified by the invariant density: $\varphi(x)$, the results of which are summarized in Table II:

The question arises whether the paradoxical behaviors just illustrated are exceptional or a generic property of ergodic processes. The positive answer is proved for a class of one-dimensional ergodic processes, to which all four processes mentioned in Example 1 belong.

Actually, a stronger and more general result can be shown as follows.

TheOREM 2 Let $\theta$ be an one-dimensional ergodic dynamical process defined on a domain $I=[a, b]$. If there exists a unique dividing point $\bar{x}$ in the interior of the interval I such that,

$$
\theta(x) \gtreqless x \text { for } x \lesseqgtr \bar{x},
$$

then for any functions $f \in C^{1}$ and $g \in C^{1}$ that are monotonic and finite in $I$, the following inequalities hold:

$$
\langle f(x) g(\theta(x))\rangle \lesseqgtr\langle f(x) g(x)\rangle, \quad \text { if } f^{\prime} g^{\prime} \gtreqless 0 .
$$



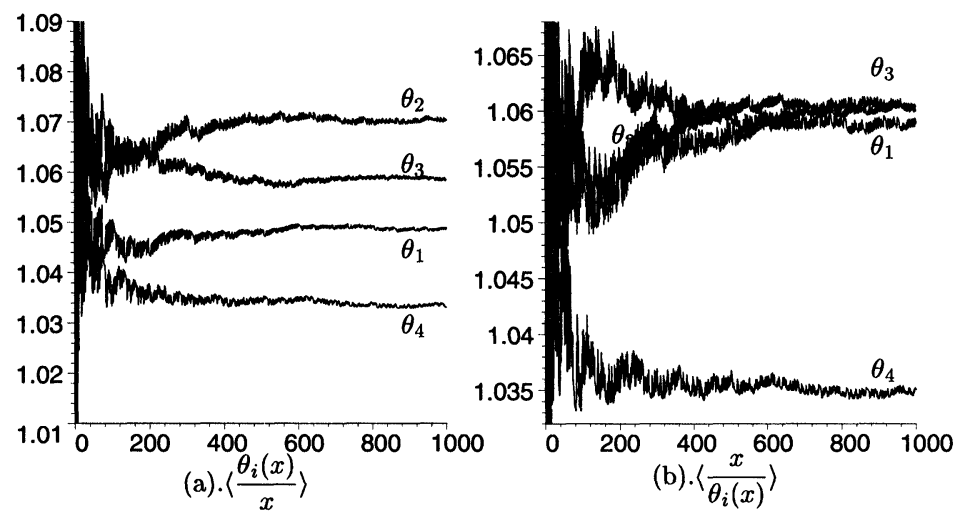

FIGURE 4 Computer simulations II.

TABLE II Illustration of inequality (13)

\begin{tabular}{lcccc}
\hline$i$ & 1 & 2 & 3 & 4 \\
\hline $\int_{I}\left(\theta_{i}(x) / x\right) \varphi(x) d x$ & 1.0503 & 1.0711 & 1.0589 & 1.0336 \\
$\int_{I}\left(x / \theta_{i}(x)\right) \varphi(x) d x$ & 1.0607 & 1.0607 & 1.0607 & 1.0348 \\
\hline
\end{tabular}

Proof Let $\varphi(x)$ be the ergodic invariant density function of $\theta$. Due to the ergodicity, the time average should be equal to the space average, so

$$
\begin{gathered}
\langle f(x) g(\theta(x))\rangle=\int_{a}^{b} f(x) g(\theta(x)) \varphi(x) d x \\
\langle f(x) g(x)\rangle=\int_{a}^{b} f(x) g(x) \varphi(x) d x \\
\langle f(x) g(\theta(x))\rangle-\langle f(x) g(x)\rangle \\
=\int_{a}^{\bar{x}} f(x)[g(\theta(x))-g(x)] \varphi(x) d x \\
\quad+\int_{\bar{x}}^{b} f(x)[g(\theta(x))-g(x)] \varphi(x) d x
\end{gathered}
$$

Referring to Figure 1, the existence of a unique dividing point $\bar{x}$ guarantees that $\theta(x) x$ for $x \lesseqgtr \bar{x}$.

We start with the case that both $f$ and $g$ are monotonic increasing functions of $x$, i.e., $f^{\prime}(x)>0$ and $g^{\prime}(x)>0$, for all $x$ :

$$
\begin{aligned}
& \int_{a}^{\bar{x}} f(x)[g(\theta(x))-g(x)] \varphi(x) d x \\
& \leq \int_{a}^{\bar{x}} f(\bar{x})[g(\theta(x))-g(x)] \varphi(x) d x, \\
& \int_{\bar{x}}^{b} f(x)[g(\theta(x))-g(x)] \varphi(x) d x \\
& \leq \int_{\bar{x}}^{b} f(\bar{x})[g(\theta(x))-g(x)] \varphi(x) d x .
\end{aligned}
$$

Therefore,

$$
\begin{aligned}
\langle f(x) g(\theta(x))\rangle-\langle f(x) g(x)\rangle \\
\leq \int_{a}^{\bar{x}} f(\bar{x})[g(\theta(x))-g(x)] \varphi(x) d x \\
\quad+\int_{\bar{x}}^{b} f(\bar{x})[g(\theta(x))-g(x)] \varphi(x) d x \\
=f(\bar{x}) \int_{a}^{b}(g(\theta(x))-g(x)) \varphi(x) d x \\
=f(\bar{x})(\langle g(\theta(x)\rangle-\langle g(x)\rangle)=0 .
\end{aligned}
$$

Here we utilize the fact that $\langle g(\theta)\rangle=\langle g(x)\rangle$.

The rest cases with different signs of derivatives of $f$ and $g$ follow the same principle.

Q.E.D

The following proposition concluded directly from Theorem 2.

Proposition 3 For any one-dimensional ergodic process $\theta$ that satisfies the condition specified in Theorem 1, the following inequalities hold for any positive integer $m$ and $n$.

$$
\begin{gathered}
\left\langle x^{m}(\theta(x))^{n}\right\rangle<\left\langle x^{m+n}\right\rangle, \\
\left\langle\frac{1}{x^{m}(\theta(x))^{n}}\right\rangle<\left\langle\frac{1}{x^{m+n}}\right\rangle \\
\quad \text { if } \theta(x) \neq 0 \text { for all } x \in I \text { and } 0 \notin I .
\end{gathered}
$$




$$
\begin{gathered}
\left\langle\frac{(\theta(x))^{m}}{x^{n}}\right\rangle>\left\langle x^{m-n}\right\rangle, \quad \text { if } 0 \notin I, \\
\left\langle\frac{x^{m}}{(\theta(x))^{n}}\right\rangle>\left\langle x^{m-n}\right\rangle, \quad \text { if } \theta(x) \neq 0 \text { for all } x \in I .
\end{gathered}
$$

Inequalities (13) then follows directly for $m=n=1$.

Although the result stated in our theorem are restricted to "one-turn" type of dynamical processes, they actually cover almost all one-humped continuous processes so far discussed in the economic literature.

Due to the fact that, if a property holds for an ergodic process $\theta$, it also holds for its iterates $\theta^{k}$, $k>1$. The latter, however, is multiple-humped. So it would be expected that the inequality (15) holds for quite a broader class of dynamical processes.

Example 2 Consider a piecewise linear process defined on the unit interval $I=[0,1]$ :

$$
T_{\sigma}(x)= \begin{cases}\sigma x & \text { if } x<1 / \sigma \\ (1 /(\sigma-1))(\sigma x-1) & \text { otherwise }\end{cases}
$$

where $\sigma>1$. The process is illustrated in Figure 5(a) and satisfies the "above-below" condition. Suppose that $f(x)=\mathrm{e}^{x}$ and $g(x)=\mathrm{e}^{-x}$, it follows $\left\langle f(x) g\left(T_{\sigma}(x)\right)\right\rangle>1$ and $\left\langle f\left(T_{\sigma}(x)\right) g(x)\right\rangle>1$, for $f^{\prime}>0$ and $g^{\prime}<0$.
Actually, $T_{\sigma}(x)$ possesses a constant invariant density $\varphi(x)=1 \chi_{[0,1]}$ that is independent of the parameter $\sigma$. Therefore, we have

$$
\begin{aligned}
\left\langle f(x) g\left(T_{\sigma}(x)\right)\right\rangle= & \int_{0}^{1 / \sigma} \mathrm{e}^{x-\sigma x} d x \\
& +\int_{1 / \sigma}^{1} \mathrm{e}^{x-((\sigma x-1) /(\sigma-1))} d x \\
= & \frac{1-\mathrm{e}^{-((\sigma-1) / \sigma)}}{\sigma-1}+\left(\mathrm{e}^{(1 / \sigma)}-1\right)(\sigma-1) \\
\left\langle f\left(T_{\sigma}(x)\right) g(x)\right\rangle= & \int_{0}^{1 / \sigma} \mathrm{e}^{\sigma x-x} d x \\
& +\int_{1 / \sigma}^{1} \mathrm{e}^{((\sigma x-1) /(\sigma-1))-x} d x \\
= & \frac{\mathrm{e}^{((\sigma-1) / \sigma)}-1}{\sigma-1}-\left(\mathrm{e}^{-(1 / \sigma)}-1\right)(\sigma-1) .
\end{aligned}
$$

The graphs of $\left\langle f(x) g\left(T_{\sigma}(x)\right)\right\rangle$ and $\left.f\left(T_{\sigma}(x)\right) g(x)\right\rangle$ calculated from (21) and (22) with respect to $\sigma$ are given in Figure 5(b). Both of them are shown to be greater than unity, which are consistent to our expectations. Due to the space limits, computer simulations are omitted.

The significance of statistical inequalities provided in this paper are yet to be explored. We provide a direct but interesting application in Walras' tatonnement process as an example.
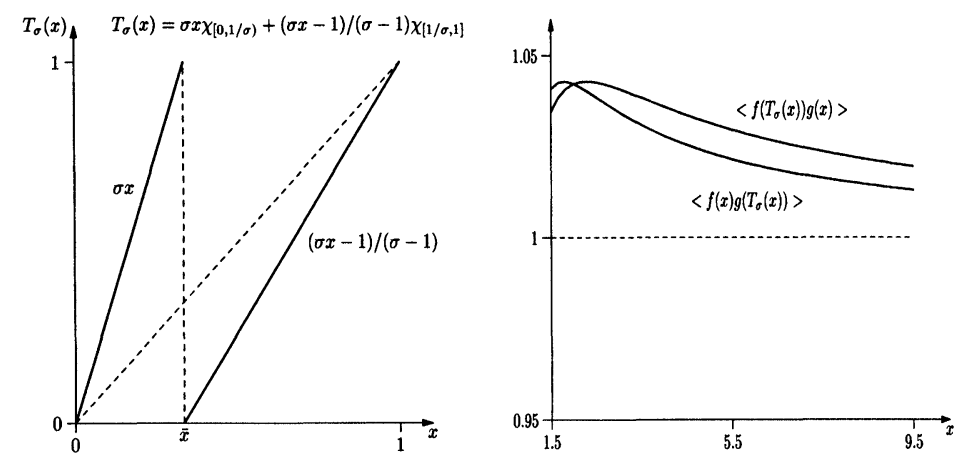

FIGURE 5 Piecewise linear map; (a) Piecewise linear process; (b) Illustration of time-averages. 


\section{ECONOMIC APPLICATION: TATONNEMENT PROCESS}

Let $S(p), D(p)$ be supply and demand functions for a given commodity with price $p$, such that $D^{\prime}(p)<0$, and $S^{\prime}(p)>0$, for any $p>0$. The market excess demand is defined by $z(p)=D(p)-S(p)$.

By tatonnement, we mean the following price adjusting process:

$$
\begin{gathered}
p_{t+1}=\theta\left(p_{t}\right)=p_{t}+\lambda z\left(p_{t}\right) \\
=p_{t}+\lambda\left(D\left(p_{t}\right)-S\left(p_{t}\right)\right),
\end{gathered}
$$

where $\lambda$ is a constant reflecting the adjustment speed.

Under conventional assumptions that $D^{\prime}\left(p_{t}\right)<$ $0, D^{\prime \prime}\left(p_{t}\right) \geq 0$ and $S^{\prime}\left(p_{t}\right)>0, \quad S^{\prime \prime}\left(p_{t}\right) \leq 0, \theta\left(p_{t}\right)$ often appears as a U-shaped curve as depicted in Figure $6 \mathrm{~b}$. Such process is apt to induce chaos as shown by Day (1994), so there exist situations that Tatonnement process specified is a chaotic and ergodic process.

When $\theta$ is ergodic, statistical characteristics such as time average exist. Proposition 1 reveals that $\langle p \theta(p)\rangle<\left\langle p^{2}\right\rangle$, which is equivalent to $\left\langle p^{2}\right\rangle+\lambda\langle p z(p)\rangle<\left\langle p^{2}\right\rangle$. Therefore, we have

$$
\langle p z(p)\rangle<0
$$

The inequality (25) resembles to the famous Walras' law in the static general equilibrium

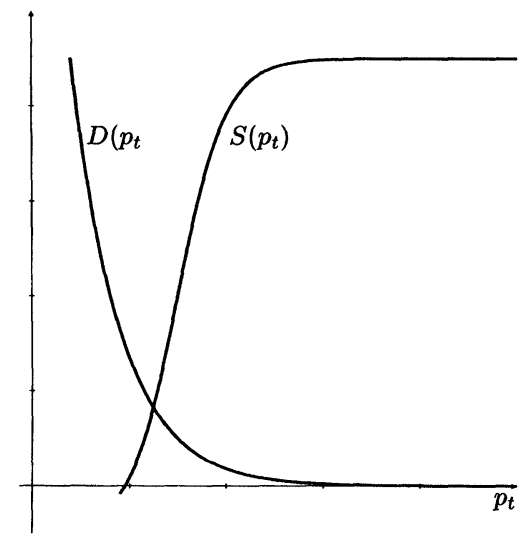

(a).Demand and Supply

FIGURE 6 Tatonnement process.

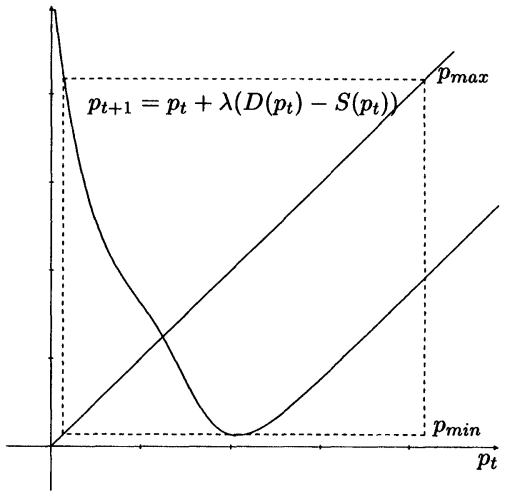

(b).Tatonnement Process
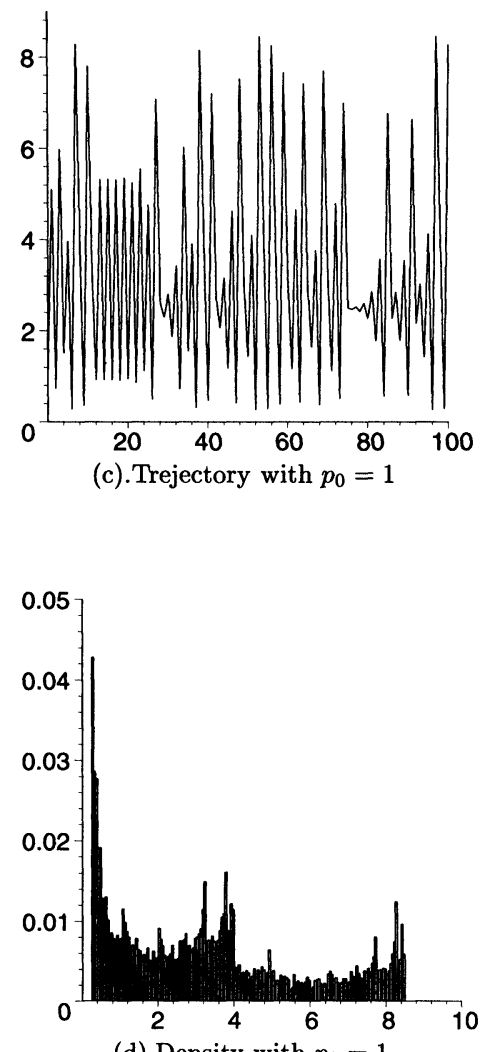

(d).Density with $p_{0}=1$

FIGURE 6 (Continued).

analysis, which states that the identity $\mathbf{P} \cdot Z(\mathbf{P})=0$ always holds true for all $\mathbf{P}$ (regardless whether the economy is in the equilibrium state or not), where $\mathbf{P}=\left(p_{1}, p_{2}, \ldots, p_{m}\right)$ is a price vector and $Z(\mathbf{P})$ the excess demand vector for $m$ commodities. 


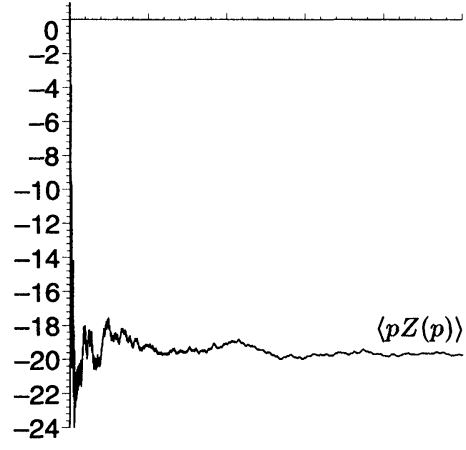

$(\mathrm{e}) \cdot\langle p Z(p)\rangle$

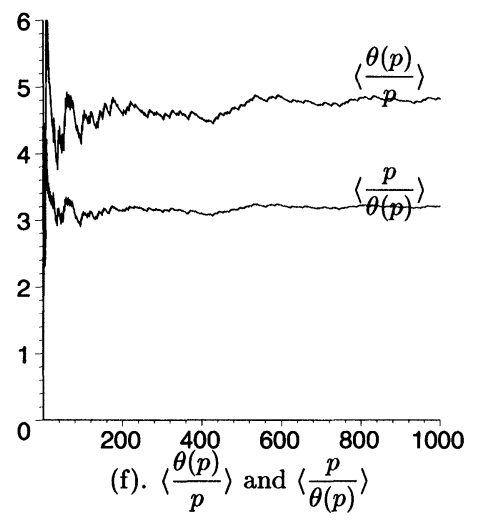

FIGURE 6 (Continued).

In term of the dynamical process defined by Eq. (24), if the tatonnement process converges to a stationary equilibrium $\tilde{p}$, we always have $z(\tilde{p})=0$ and so $\tilde{p} z(\tilde{p})=0$. But if the process is chaotic and ergodic, then in the long-run, its average $\langle p z(p)\rangle$ is negative in general. So the inequality $(25)$ provides further insight about long-run dynamical characteristics of an ergodic tatonnement process, and hence will be referred to as dynamical Walras law.

Define the inflation rate as

$$
\pi_{t}=\frac{p_{t+1}-p_{t}}{p_{t}} .
$$

Since $\theta\left(p_{t}\right)$ satisfies the conditions specified in Theorem 2, we therefore conclude that the average inflation rate is always positive.

Example 3 (Huang, 1999) Let $D\left(p_{t}\right)=a \mathrm{e}^{-\left(p_{t}-h\right)}$ and $S\left(p_{t}\right)=c \mathbf{e}^{b\left(p_{t}-h\right)}-d$, where $a, b, c, d$, and $h$ are positive constants. Figure 6(a) illustrates their graphs with $a=1, h=3, b=2, d=1$ and $c=10$.

The tatonnement process defined by Eq. (23) with $\lambda=0.5$ is shown in Figure 6(b).

For a fixed initial price $p_{0}=1$, computer simulations have been performed and presented in Figure 6(c) for trajectory of first 100 iterations, Figure 6(d) for simulated invariant density, Figure 6(e) for average $\langle p z(p)\rangle$, and Figure 6(f) for average $\langle(\theta(p) / p)\rangle$ and $\langle(p / \theta(p))\rangle$, respectively.

\section{CONCLUSION}

Some interesting statistical inequalities of ergodic dynamical process are discussed and illustrated with computer simulations. It is shown that, for a broader class of one-dimensional discrete chaotic process, the average growth rate is always positive. Future research will directed to whether such property is shared by all chaotic and/or ergodic processes, and to the necessary or sufficient conditions for its validity in high-dimensional processes.

\section{References}

Day, R. H. and Pianigiani, G. (1991) Statistical Dynamics and Economics, Journal of Economic Behavior and Organization, 16(1-2), 37-83.

Day, R. H. and Shafer, W. (1987) Ergodic Fluctuations in Deterministic Economic Models, Journal of Economic Behavior and Organization, 8, 57-76.

Day, R. H. (1994) Complex Economic Dynamics. Volume 1. MIT Press. (Cambridge and London).

Huang, W. (1989) Distributional Dynamics of Economic Systems, Ph.D Dissertation, University of Southern California.

Huang, W. and Day, R. H. (1993) Distributional Dynamics of Bull and Bear Markets, In: Day, R. H. and Chen, P. (Eds.) Evolutionary Dynamics and Nonlinear Economics, Oxford University Press.

Huang, W. (1999) Statistical Dynamics of Discrete Tatonnement Process, Working Paper, Nanyang Technological University.

Lasota, A. and Mackey, M. C. (1985) Probabilistic Properties of Deterministic Systems, Cambridge University Press.

Lorenz, E. N. (1993) The Essence of Chaos., University of Washington.

Medio, A. (1999) Nonlinear Dynamics and Chaos Part II: Ergodic Approach, Macroeconomic Dynamics, 3, 84-114.

Sigmund, K. (1992) Time Averages of Unpredictable Orbits of Deterministic Systems, Annals of Operation Research, 37, $217-218$ 


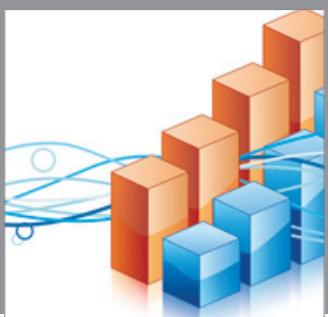

Advances in

Operations Research

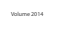

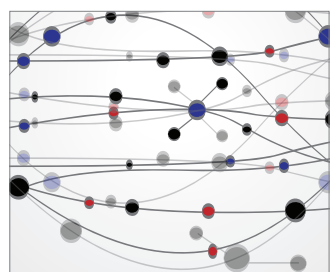

\section{The Scientific} World Journal
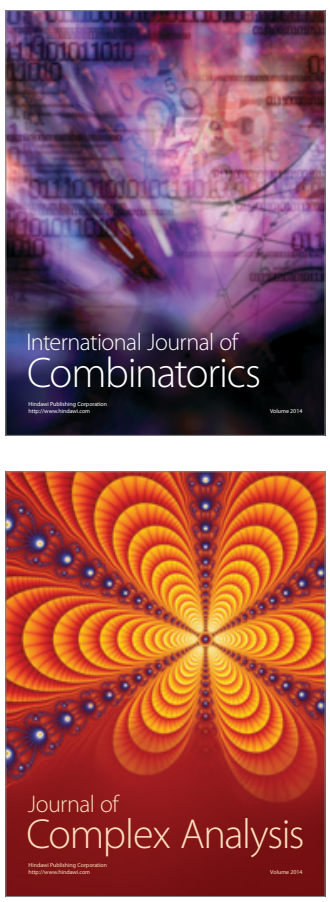

International Journal of

Mathematics and

Mathematical

Sciences
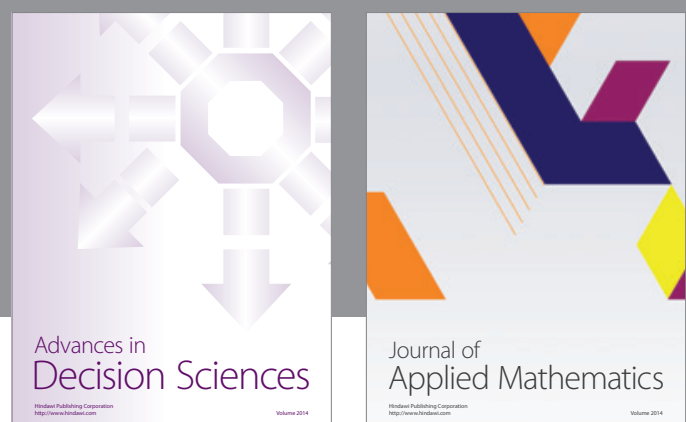

Journal of

Applied Mathematics
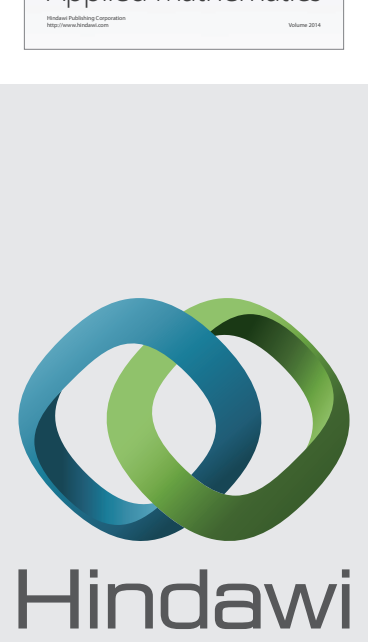

Submit your manuscripts at http://www.hindawi.com
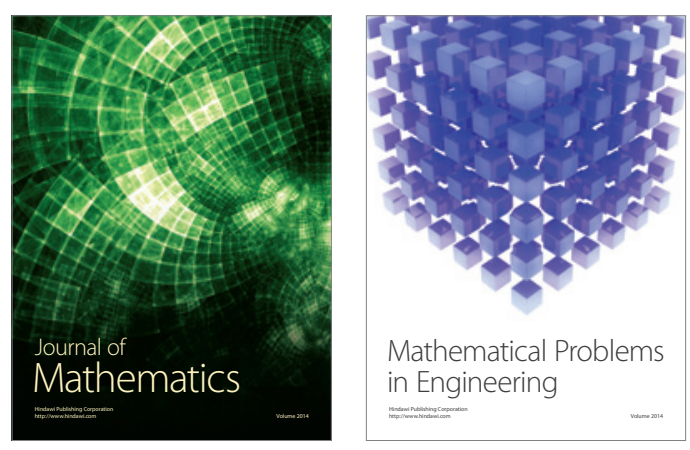

Mathematical Problems in Engineering
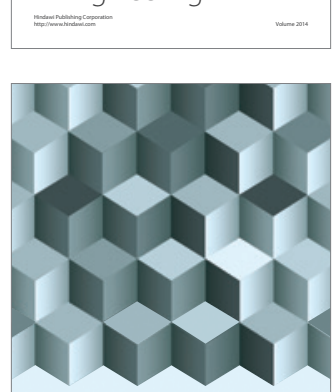

Journal of

Function Spaces
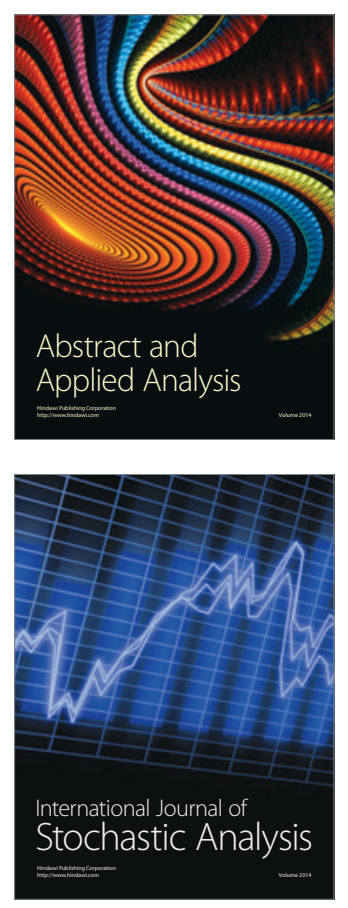

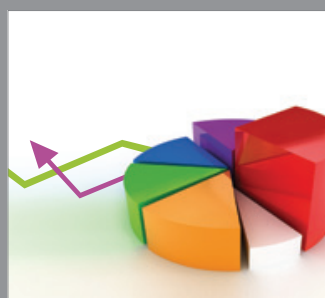

ournal of

Probability and Statistics

Promensencen
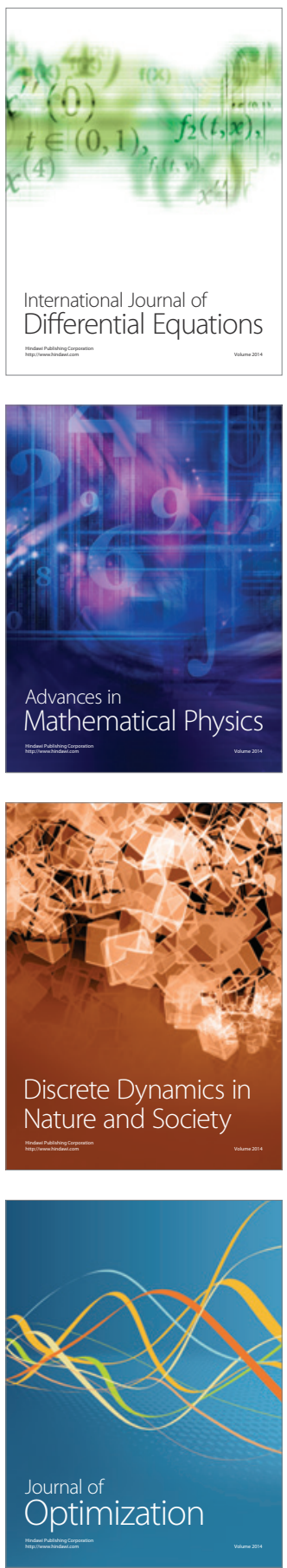